\title{
Academic ebooks and the mobile user experience
}

\author{
Mark Williams and Ben Showers
}

Jisc Collections and Jisc

How did you go bankrupt?

Two ways. Gradually, then suddenly.

Ernest Hemingway, The Sun Also Rises

These two trajectories - gradual, almost imperceptible change and then sudden, rapid transition - define much of the technological change that has confronted universities and colleges over the past few decades. While this progression is not unique to academia, it does seem particularly marked in the academic sector, from the initial caution shown to the web and the lack of institutional presence online to the experimentation with mobile learning, which remains experimental and largely peripheral. Serious change always seems such a long way off, until it changes everything.

In contrast to the development of the Kindle and iPad and the associated content platforms of Amazon and iBooks, the "revolution" in digital academic content seems to be indefinitely waiting in the wings. Much of the innovation in digital academic content has been in academic ejournals which have for a number of decades cemented themselves as the primary mode of delivery for

How to cite this book chapter:

Williams, M. and Showers, B. 2014. Academic ebooks and the mobile user experience. In: Woodward, H. (ed.) Ebooks in Education: Realising the Vision. Pp. 51-58. London: Ubiquity Press. DOI: http://dx.doi.org/10.5334/bal.f 
scholarly articles. With increasing rapidity, however, students' and researchers' changing requirements and expectations mean that they expect an intuitive and mobile platform approach to the discovery of, and access to, ebooks and digital content. Academic users are no longer willing to make exceptions for scholarly content.

In this chapter we make an attempt to explore the changing requirements of students and researchers regarding access to scholarly digital content, and the challenges and implications for content and service providers in adapting to these changing expectations. Primarily, there is a focus on the consumption of ebooks and digital content by users, ensuring that institutions and publishers take a user-centric perspective in the ways digital content is delivered and used. It also provides an opportunity to explore the particular value of ebooks for the scholarly enterprise. They offer a way to enhance the experience of engaging with the texts themselves - access to the underlying data, interactive images, annotation functionality, and so on. There is also the potential for new models and approaches to inform the research process itself - being able to access the content in new ways, such as text and data mining, for example.

Fundamentally, this chapter is an attempt to move academic ebooks beyond the current paper (pdf) archetype and towards something which exploits the inherent potential of digital content. Ebooks deliver a user experience that exceeds the expectations of students, researchers and users.

\section{The landscape}

Plenty of reports on the web detail trends of mobile versus desktop usage and, although such reports may differ in terms of the timescales they predict, they mostly agree that mobile usage will exceed desktop very soon. ${ }^{1}$ The fact that we no longer concern ourselves with statistics about smartphone sales versus feature phones shows how de-facto web consumption on a mobile device has become. The one distinction worth making is between the use of mobile tablets and the use of smartphones, although even this distinction loses relevance outside of conversations around content usability and authentication.

It is in this mobile ecosystem that academic ebooks have to exist. Even in the traditional desktop scenario, where publishers and institutions have tinkered with the ebook platforms and content to fit a reasonably consistent template, there is significant variability among the products, something that institutional librarians have had to take into account when developing their user guides. ${ }^{2}$ Once the huge variety of mobile devices and operating systems is introduced into the equation, the consistency of the user experience can be severely compromised.

\footnotetext{
${ }^{1}$ http://www.gartner.com/newsroom/id/2645115

2 http://adat.crl.edu/ebooks
} 
It is user expectation that impacts the actual definition of what an ebook is. Users who are used to having a straightforward consistent experience when using an individual, commercially purchased ebook for their own leisure enjoyment are often confronted with a completely different experience with an academic ebook in a mobile context. So much so, one wonders whether the same definition of an ebook really applies to the two scenarios. Depending on the ebook provider, a user won't be able to read the book anytime, anywhere on their device, nor will they be able to lend the book, to underline passages, or to save the entire book. They may not even be able to open the book because of authentication issues, in which case the question needs to be asked: Do they even have in their virtual possession, a book?

It is this gap in user experience that causes consumption of an ebook on a mobile device to fall behind other experiences. Academic ebook provider platforms differ to such a degree that institutions are forced to develop bespoke instructions on their use, something which would be anathema to a large scale retailer of commercial ebooks - and something which certainly defies current web design best practice of intuitive interaction.

The equation is admittedly more complex than at first glance. An ebook is not simply a single file. Effectively it is the sum of a publisher platform. It is a file that holds content and supports discovery, a content file ideally developed to common standards such as $\mathrm{EPUB}^{3}$. In addition to this, there may be reader software such as Adobe Editions wrapped around the platform, and beyond this, there may also be variety of authentication procedures attached. All of these elements then have to operate on a variety of operating systems and devices. The sum of this complex relationship is greater than the challenge of any individual element.

Because of this complexity, the presentation of ebooks within an app in the mobile environment becomes very tempting. In an app, the developer has far greater control over the processes occurring, which should lend itself to a more bug-free and issue-free user experience. The tendency by publishers to focus on apps for mobile delivery can be attributed to both demand - after all, this is the way in which many users consume other content on their devices - and the fact that half the mobile tablet space is essentially app-driven by design, such as the iPad. So app deployment on smartphones can remove some of the usability issues discussed here - issues with the platform, such as discovery and download and with the content, and readability on a three-inch screen.

But app-driven ebooks pose significant authentication challenges. Many apps use device twinning, in which a user first authenticates to a platform in a traditional web environment, often using IP or federated access, and then requests a code that is then sent by email. They can then use this to authenticate their mobile device. It is a pragmatic solution, but one that in many ways

\footnotetext{
${ }^{3}$ http://idpf.org/epub/30
} 
undermines the UK's current academic licensing models and authentication systems. By receiving a key code and twinning their account, the user is effectively authenticating the phone but not the user. If the phone is lost or stolen, or simply borrowed, access is often possible for periods of up to 90 days there is no industry standard here. Only with the use of two-factor authentication or the use of activation lock software, making the user enable password protection on the phone, is this vulnerability mitigated. However, there is an ironic twist here. For the user to enable twinning and receive an initial authentication code to enable them to access their resource while mobile, they frequently need to be on campus in the first instance. They are required to be within the designated IP range, or to navigate a federated login on a very small screen, which is of course the very issue they are trying to avoid by enabling device twinning.

Device twinning is, however, still one of the more pragmatic solutions from the user's point of view. However, authentication can be far more complex. A user will often have to juggle logins for an app store, Adobe Digital Editions, a federated access account and a social ID just to access the same piece of content on a range of devices. There is often further difficulty arising from the policies of the user's own institution, if social web apps such as Facebook have been blocked. On a small-size mobile device screen, those additional obstacles can become unmanageable. The effects of a single bug or problem encountered by the user become multiplied as the number of processes necessary for a user to gain access to and read an ebook increases.

Fortunately, the additional tools that a user frequently has to download in order to read an ebook successfully, such as Adobe Editions, can at least be installed on a user's mobile device by the user themselves. But in a lockeddown institutional desktop environment that may well be impossible. This again leads to users having a very different and inconsistent experience when accessing the same content on different platforms.

Consistency of experience is a must, with an expectation that users on a mobile device will require offline access, will be able to annotate on and offline, will be able to cut and paste according to the agreed licence, and have a flowing layout that is readable on and adapts to the full range of devices.

Providers of commercial mainstream content have learned the lessons that making content difficult to access can lead to users exploring alternative ways of getting that content. If an institutional user illegally streams a resource for which their institution already holds a licence, this tells us that it is not a question of affordability but one of usability. Platforms such as Adobe Digital Editions do offer ebook providers the ability to set up borrowing rather than purchase ebook offerings, but at a cost of ease of use. A poor first-time experience on an ebook platform for a user may well be their last as they explore other resources. This should be considered by publishers as they make choices as to what to protect through Digital Rights Management (DRM) and what not to protect. 
Feedback from institutions also demonstrates that ebook performance on mobile devices is considerably "buggier" than in any other environment. Clearly, the range and limitations of the browsers, platforms and devices in question are larger than the typical desktop experience. This makes it harder to design and code for, and consequently requires more effort put into testing to allow for the range of devices to be used with the content. Simply emulating generic mobile experiences is not sufficient for this, and if it takes more resources to ensure an ebook product is properly tested and fit for mobile purpose, then that resourcing should be provided, rather than providing an experience that may just dishearten and drive users away. Additional associated costs should be worked into the ebook model.

Some studies have shown that many users prefer not to read ebooks on mobile devices, although the important question is - why? ${ }^{4}$ If the discovery and delivery phase is so difficult, then this will surely cloud the user experience of the actual consumption of content. A reflexive answer that small screens are an issue for the user avoids confronting this, and flies in the face of the trend for larger mobile device screens. While we need to do more work on finding out what users want to use mobile devices for, we need to remove what barriers we can, and ensure that accessing ebooks on a mobile device is seen not just as added value, but as a core component of a licence and user experience of that resource.

\section{Meeting user needs with academic ebooks}

Removing the barriers for the enduser is essential if content and service providers are going to able to meet the expectations and requirements of our users. If we fail to remove this friction for the users, they will simply adopt the path of least resistance, finding easier, and possibly less trusted and secure, ways to find and access the content they need.

There have been over the past few years a number of efforts to begin collaboration and sharing of best practice and exemplars in the online and mobile delivery of ebooks and digital content. Some of these, like the Library Success $\mathrm{Wiki}^{5}$ and the Mobile Technologies in Libraries blog ${ }^{6}$ are collaborative, community attempts to impose some order on the disparate and fragmented mobile content environment in universities and colleges. Building on this work, Jisc, along with a group of academic librarians ${ }^{7}$, hosted two workshops to see whether it was possible for the community (libraries, publishers,

\footnotetext{
${ }^{4}$ http://blogs.lse.ac.uk/impactofsocialsciences/2013/06/11/what-do-academics-want-asurvey-of-behaviours-and-attitudes-in-uk-higher-education/

${ }^{5}$ http://www.libsuccess.org/M-Libraries

${ }^{6}$ http://mlibraries.jiscinvolve.org

7 http://mlibraries.jiscinvolve.org/wp/2013/07/25/jisc-collection-mobile-issues-workshop/
} 
aggregators and systems vendors) to come together to clarify the ways they could meet user needs and overcome the shared challenges in achieving this. As a result of these workshops there has emerged what has been dubbed a "mobile manifesto" for improving mobile services to students and researchers.

\section{A mobile manifesto for ebooks}

To ensure that libraries and publishers can deliver the kinds of content and services that students and researchers expect, the manifesto aims to provide concrete activities that will fulfil the core requirements that emerged from the library and publisher workshops. These include a number of specific statements of intent, including:

\section{Authoritative record of relevant mobile products}

The proliferation of mobile resources and devices makes testing and assessment of resources an onerous task for any individual library or organisation. A crowd-sourced list, or record, has the advantages of spreading the workload and developing consensus on user experience. Libraries and publishers should develop this partnership and the process and ongoing end results need to be effectively surfaced for the community.

\section{A mobile standard}

While not quite a fully-fledged standard, this would be a checklist against which the functionality of a mobile resource can be measured. The work done by the Book Industry Study Group on the EPUB support grid ${ }^{8}$ and the EPUB3 standard (which is designed for reflowable content) provides a model for how this might work. Librarians would be able to see instantly the kinds of functionality they could expect from a particular mobile resource and be able to advise users on what they can expect to be able to do with it.

\section{Product roadmap/status updates}

There is a clear need for a grading system for mobile products that express the status of the development. For example, grades may include whether a product is in trial or will have a continuous upgrade path. This could form part of the authoritative record of mobile products already discussed in section 1 above.

\footnotetext{
${ }^{8}$ https://www.bisg.org/epub-3-support-grid
} 


\section{Accessibility and compatibility}

Any mobile development should enable content to be accessed easily and read on all mobile devices, using clear flowing text. This should meet not just WC3 accessibility standards ${ }^{9}$ but also general ease of use requirements for all users accessing via mobile devices. Accessibility of content on mobile devices benefits everyone. Similarly, cross-platform compatibility is required to ensure that content and resources can be seamlessly accessed on different mobile devices. Users should be able to start reading an article on their desktop and pick up where they left off on their iPad as they travel home on the bus.

\section{Access and authentication}

Accessing content should be made as simple and intuitive for the user as possible. There are only a certain number of access scenarios, and all publishers should have the same common requirement of achieving secure authentication to their resources. This can be done through collaboration between libraries and publishers, and agreement and consolidation around agreed standards based on models previously discussed.

The manifesto is primarily a way to surface the shared challenges and concerns of the community in providing access to ebooks for its users and customers. It also provides a locus for discussion of these issues and a common source for exemplars and best practice to be shared and updated, both nationally and internationally.

Fundamentally, it is the beginning of the necessary collaboration between all the stakeholders involved in ebook creation and provision. They can begin working together to tackle the challenges that must be overcome to provide users with a positive mobile experience. It also marks the start in further work Jisc and the library partners would like to do to begin taking some concrete action in the mobile ebook space.

\section{Conclusion}

This chapter has highlighted some of the major challenges that currently confront institutions, libraries, publishers and other stakeholders in the scholarly content supply chain in providing mobile access to those resources. Taken together, these challenges form a systemic and "hard" problem; a problem which requires work on multiple fronts and in partnership and collaboration with others. It is not easy to overestimate the scale of the problem that is faced in delivering a mobile experience that students and researchers would expect when accessing and using scholarly ebooks.

${ }^{9}$ http://www.w3.org/standards/webdesign/accessibility 
But progress is being made, and while there are a number of initiatives from the stakeholders involved, there is also a recognition that many of these problems cannot be solved without collaboration. And the need to solve these issues is a priority.

Overwhelmingly, the message is that we must begin to act. While change so far for academic content has been gradual, this will not be the norm. The pace of change is going to increase, user expectations will change in relation to these new developments, and the technology we are beginning to adapt to will disappear to be replaced with something else. If we cannot meet the needs of our users while the pressures on academic content remain relatively benign, then the inevitable suddenness of future change threatens to overwhelm our capacity to provide content and services at all. 